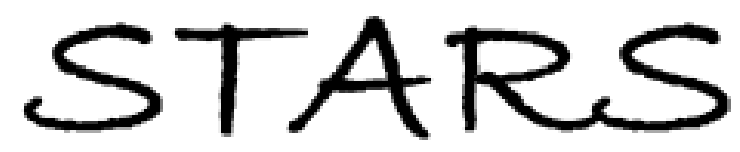

University of Central Florida

STARS

$1-1-2005$

\title{
Design, fabrication, and characterization of antenna-coupled metal-oxide-metal diodes for dual-band detection
}

\author{
Mohamed R. Abdel-Rahman \\ Brian Monacelli \\ Arthur R. Weeks \\ Guy Zummo \\ Glenn D. Boreman \\ University of Central Florida
}

Find similar works at: https://stars.library.ucf.edu/facultybib2000

University of Central Florida Libraries http://library.ucf.edu

This Article is brought to you for free and open access by the Faculty Bibliography at STARS. It has been accepted for inclusion in Faculty Bibliography 2000 s by an authorized administrator of STARS. For more information, please contactSTARS@ucf.edu.

\section{Recommended Citation}

Abdel-Rahman, Mohamed R.; Monacelli, Brian; Weeks, Arthur R.; Zummo, Guy; and Boreman, Glenn D., "Design, fabrication, and characterization of antenna-coupled metal-oxide-metal diodes for dual-band detection" (2005). Faculty Bibliography 2000s. 4934.

https://stars.library.ucf.edu/facultybib2000/4934

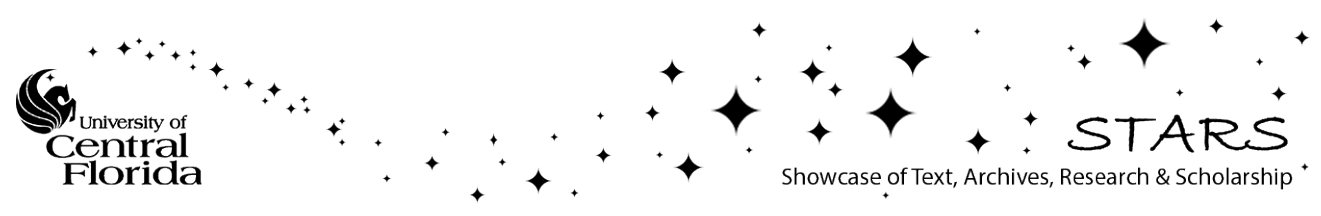




\section{Design, fabrication, and characterization of antenna-coupled metal-oxide-metal diodes for dual-band detection}

\author{
Mohamed R. Abdel-Rahman, MEMBER SPIE \\ Brian Monacelli, MEMBER SPIE \\ Arthur R. Weeks, MEMBER SPIE \\ Guy Zummo \\ Glenn D. Boreman, FELLOW SPIE \\ University of Central Florida \\ Infrared Systems Lab/CREOL \\ Orlando, Florida 32816 \\ E-mail: boreman@creol.ucf.edu
}

\begin{abstract}
Two designs for antenna-coupled Ni-NiO-Ni diodes are fabricated and tested for dual-band detection in the millimeter-wave (MMW), 94-GHz, and infrared (IR), 28.3-THz, frequencies. The detector noise, antenna receiving properties, and noise equivalent power (NEP) are measured. The simultaneous dual-band response is verified. () 2005 Society of Photo-Optical Instrumentation Engineers. [DOI: 10.1117/1.1929647]

Subject terms: metal-oxide-metal diodes; slot antennas; dual-band response; metal-oxide-metal diode noise.

Paper 040666R received Sep. 13, 2004; revised manuscript received Dec. 14, 2004; accepted for publication Dec. 21, 2004; published online Jun. 3, 2005. This paper is a revision of a paper presented at the SPIE Conference on Radar Sensor Technology and Passive Millimeter-Wave Imaging Technology, Apr. 2004, Orlando, FL. The paper presented there appears (unrefereed) in SPIE Proceedings Vol. 5410.
\end{abstract}

\section{Introduction}

Because of the difference in wavelengths, IR imagers offer inherently high resolution, while millimeter-wave (MMW) systems have better penetration through atmospheric aerosols such as fog and smoke. In this paper, we aim to integrate both MMW and IR detection capabilities in one sensor and thus combine the advantages of high resolution and all-weather detection capabilities. The ratio between the two desired frequencies of operation, $94 \mathrm{GHz}$ and 28.3 $\mathrm{THz}$, is roughly 300:1. Special detector design is required to accommodate the desired dual-band performance. One possible design is a frequency-independent antenna structure with a small enough feed to allow for operation at the high-frequency limit; the sensor is then to be located at the feed. Another approach, which is implemented here, is to integrate the sensor with two resonant antenna structures at the two required frequencies of operation. Antenna-coupled metal-oxide-metal (MOM) diodes have been used in the past for detection and mixing in the IR (Ref. 1) and MMW (Ref. 2) portions of the spectrum. A MOM diode with a junction capacitance low enough to rectify IR radiation will also rectify MMW radiation; thus, it is a good candidate for this application. The cutoff frequency of a MOM diode junction is given $b^{3}$

$f_{c}=\frac{1}{2 \pi R_{A} C_{D}}$,

where $R_{A}$ represents the resistance of the antenna, and $C_{D}$ is the capacitance of the diode, which is given by

$C_{D}=\frac{\epsilon_{0} \epsilon_{r} A}{d}$,

0091-3286/2005/\$22.00 @ 2005 SPIE where $\epsilon_{r}$ is the relative permittivity of the oxide, $A$ is the contact area between the two metal electrodes, and $d$ is the thickness of the oxide layer.

Due to the nonlinear relationship between the tunneling current and the applied bias voltage of a MOM junction, the antenna-coupled ac voltage $V_{\text {ac }}$ developed across the junction will be rectified resulting in a dc signal described by $^{4}$

$V_{\text {rect }}=-\frac{1}{4} \frac{I^{\prime \prime}\left(V_{\text {bias }}\right)}{I^{\prime}\left(V_{\text {bias }}\right)} V_{\text {ac }}^{2}$,

where $I^{\prime}$ and $I^{\prime \prime}$ represent the first and second derivatives evaluated at $V_{\text {bias }}$ of the current-voltage (I-V) characteristic curve of the junction. ${ }^{4}$

Sensitivity is a parameter used to characterize the performance of a MOM diode; it is proportional to the current responsivity of a MOM diode and is defined by ${ }^{4}$

$S=\frac{-I^{\prime \prime}\left(V_{\text {bias }}\right)}{I^{\prime}\left(V_{\text {bias }}\right)}$.

In this paper, two dual-band antenna coupled MOM diode designs were implemented:

1. MMW slot antenna/IR dipole antenna coupled to $\mathrm{Ni}-$ $\mathrm{NiO}-\mathrm{Ni}$ diode

2. MMW twin-slot antenna/IR slot antenna coupled to $\mathrm{Ni}-\mathrm{NiO}-\mathrm{Ni}$ diode

\section{Sensor Designs}

\subsection{Design 1: MMW Slot Antenna/IR Dipole Antenna Coupled to Ni-NiO-Ni Diode}

A MOM diode is located at the center of a MMW slot antenna and is connected to an IR dipole antenna. The device is studied in detail in Ref. 5. The length of the MMW 


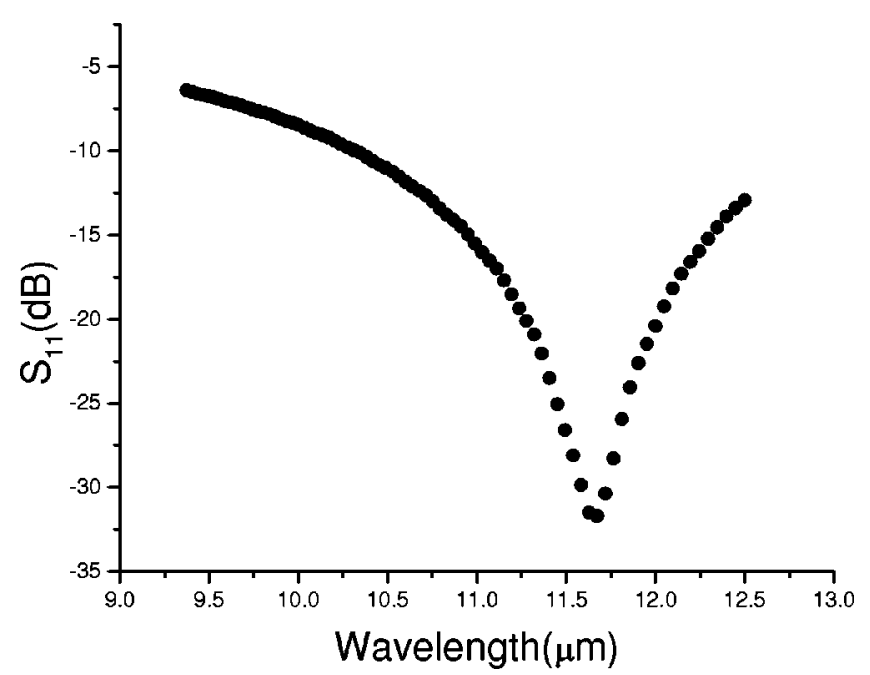

Fig. 1 Plot of $S_{11}$ versus wavelength for IR dipole antenna.

slot is $0.20 \lambda_{0}$, its width is $4.7 \times 10^{-3} \lambda_{0}$. It is designed to operate at first resonance at $92.5 \mathrm{GHz}$ and is predicted by Ref. 6 to have an impedance of $160 \Omega$. The IR dipole was designed for $\lambda / 2$ resonant operation at $28.3 \mathrm{THz}$; its total arm length is $3 \mu \mathrm{m}$ and its cross-arm width is $0.25 \mu \mathrm{m}$. IE3D (Ref. 7) was used to simulate the dipole. The reflection coefficient $S_{11}$ is plotted versus wavelength in Fig. 1 predicting a resonance at $11.6 \mu \mathrm{m}$ with a bandwidth of 1 $\mu \mathrm{m}$ and an antenna impedance of $52 \Omega$. The bandwidth is defined as the voltage standing wave ratio (VSWR) between the antenna, and the diode impedance is $\leqslant 1.5$.

The fabricated diode contact area for design 1 is 0.075 $\mu \mathrm{m}^{2}$, and the oxide layer thickness was measured to be 3.5 $\mathrm{nm}$ with a relative permittivity, determined by IR ellipsometry, of 3.24. Based on these measurements, the capacitance of the diode as defined by Eq. (2) is estimated to be $512 \mathrm{aF}$. Based on the preceding antenna impedance simulations and substituting in Eq. (1), the cutoff frequency for a $160-\Omega$ MMW slot antenna coupled to a $512-\mathrm{aF}$ MOM diode is $1.94 \mathrm{THz}$, and for a $52-\Omega$ IR slot antenna it is $5.97 \mathrm{THz}$. The device is then expected to operate above the cutoff frequency in the MMW band and below the cutoff frequency in the IR band. A smaller capacitance diode is required for the device to operate above cutoff frequency in the IR; this could be achieved by either increasing the thickness of the NiO layer or by decreasing the diode's contact area. Since tunneling currents would be significantly reduced by increasing the thickness of the oxide layer more than $3.5 \mathrm{~nm}$, decreasing the contact area is a more feasible modification.

\subsection{Design 2: MMW Twin-Slot Antenna/IR Single Slot}

The sensor configuration is shown in Fig. 2. Two MMW slot antennas are etched in a $3 \times 3-\mathrm{mm}$ Au ground plane. The optimum separation $x$ between the MMW slot antennas is determined from ${ }^{8}$

$P(x)=2\left[1+J_{0}(x)+J_{1}(x)\right]$

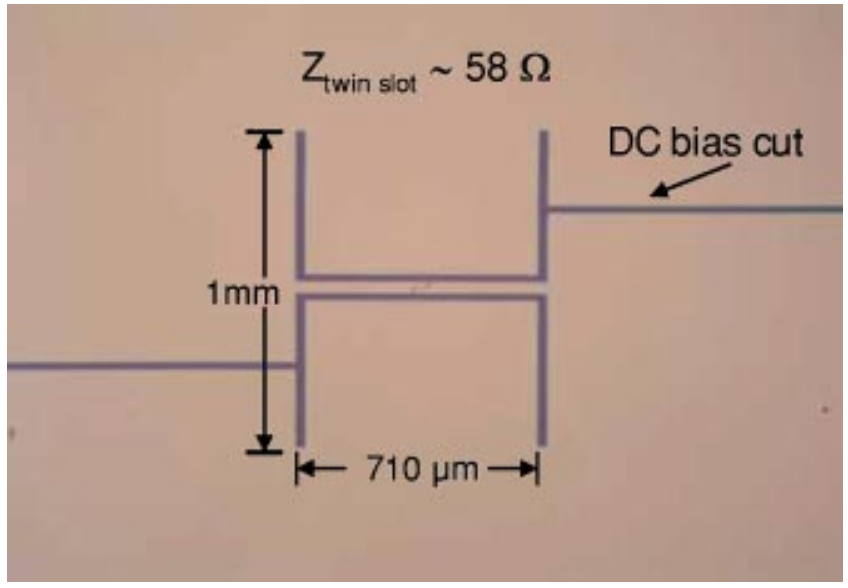

Fig. 2 CPW-fed MMW twin-slot antenna.

where $P(x)$ is the surface-wave power lost to the $\mathrm{TM}_{0}$ mode, and $J_{0}$ and $J_{1}$ are Bessel functions of the first kind of orders 0 and 1 . The optimum slot separation $x$ is determined where the power lost to the dominant $\mathrm{TM}_{0}$ mode $P(x)$ is least. The optimum slot separation $x$ is calculated to be $710 \mu \mathrm{m}\left(0.22 \lambda_{0}\right)$. A coplanar waveguide $(\mathrm{CPW})$ is utilized to feed coupled MMW radiation to the MOM sensor located at the center of the CPW. The CPW strip width is $40 \mu \mathrm{m}$, corresponding to a characteristic impedance of 51 $\Omega$. The CPW is modified as shown in Fig. 3 to accommodate an IR slot antenna. The dc cuts are made in both the MMW slot ground plane and the modified central area of the CPW to allow for sensor bias. The MOM diode is connected to the IR slot antenna as shown in Fig. 4.

The MMW slot antenna is designed to operate at second resonance based on Ref. 6, in which a twin-slot antenna having a length-to-width ratio of 40:1 on a Si substrate was predicted to have an admittance of $\sim 17 \times 10^{-3} \Omega^{-1}$, which corresponds to an impedance of $\sim 58 \Omega$. The IR slot was designed as a one-wavelength slot for operation at 28.3 THz; its length is $0.75 \lambda_{0}$ and its width is $0.085 \lambda_{0}$. The design was based ${ }^{9}$ on one-wavelength slot theory for wavelengths below $120 \mu \mathrm{m}$. The IR slot antenna was simulated by IE3D (Ref. 7); the reflection coefficient $S_{11}$ is plotted versus wavelength in Fig. 5, predicting a resonance at 11.1

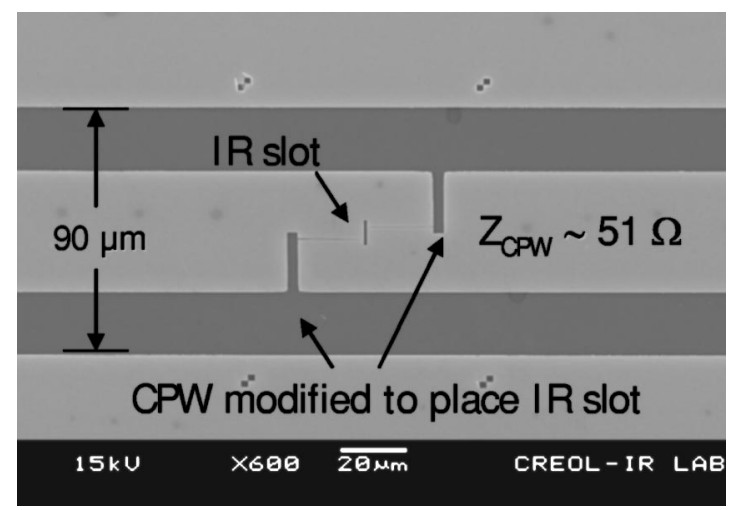

Fig. 3 Modified CPW to accommodate IR slot antenna. 


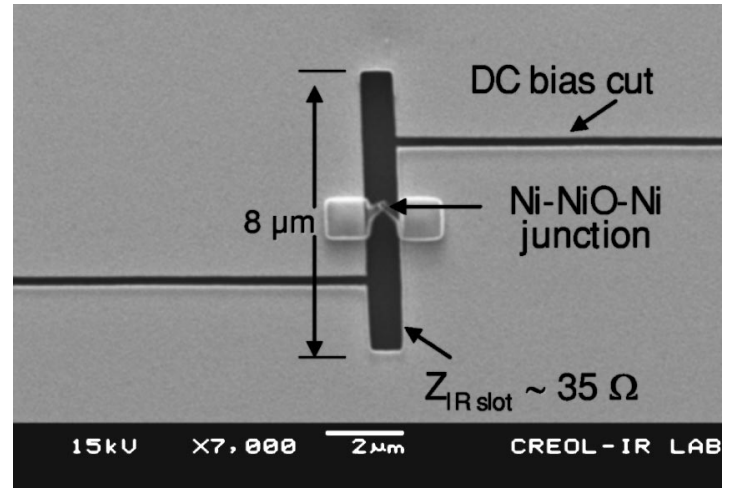

Fig. 4 IR slot coupled to Ni-NiO-Ni diode.

$\mu \mathrm{m}$ with a bandwidth of $2.4 \mu \mathrm{m}$ and an antenna impedance of $35 \Omega$.

The fabricated diode contact area for design 2 is 0.0014 $\mu \mathrm{m}^{2}$, and the oxide layer thickness was measured to be 3.5 $\mathrm{nm}$ with a relative permittivity, determined by IR ellipsometry, of 3.24. Based on these measurements, the capacitance of the diode is estimated to be $117 \mathrm{aF}$. Based on the preceding antenna impedance simulations and substituting in Eq. (1), the cutoff frequency for a 58- $\Omega$ MMW twin-slot antenna coupled to a $117 \mathrm{aF}$ MOM diode is $23 \mathrm{THz}$ and for a $35-\Omega$ IR slot antenna is $38 \mathrm{THz}$. Therefore, the device is expected to operate above the cutoff frequency in both bands.

\section{Fabrication}

\subsection{Slot Antenna Fabrication Process}

The device in design 2 is fabricated on a high resistivity $\mathrm{Si}$ substrate coated with a 500 -nm layer of $\mathrm{SiO}_{2}$ for electrical isolation. The substrate was coated with a $15-\mathrm{nm}$ layer of $\mathrm{Cr}$ and a 120-nm layer of Au. The CPW-fed twin slot structure is then patterned in electron-beam positive resist. Sputter etching in $\mathrm{rf}$ generated $\mathrm{Ar}^{+}$plasma was used to etch the slots into the $\mathrm{Au}$ ground plane utilizing PMMA as a mask.

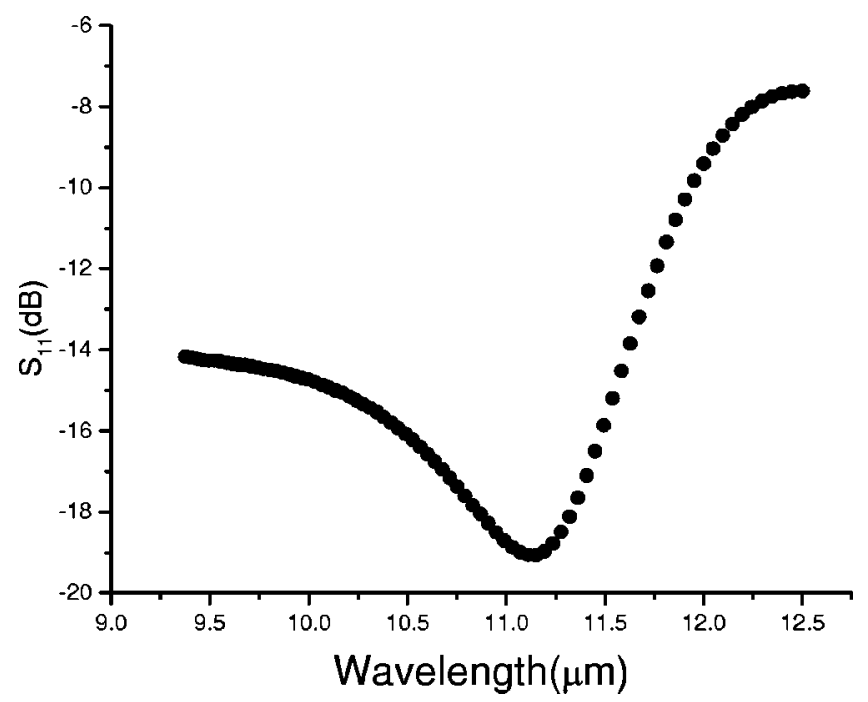

Fig. 5 Plot of $S_{11}$ versus wavelength for IR slot antenna.

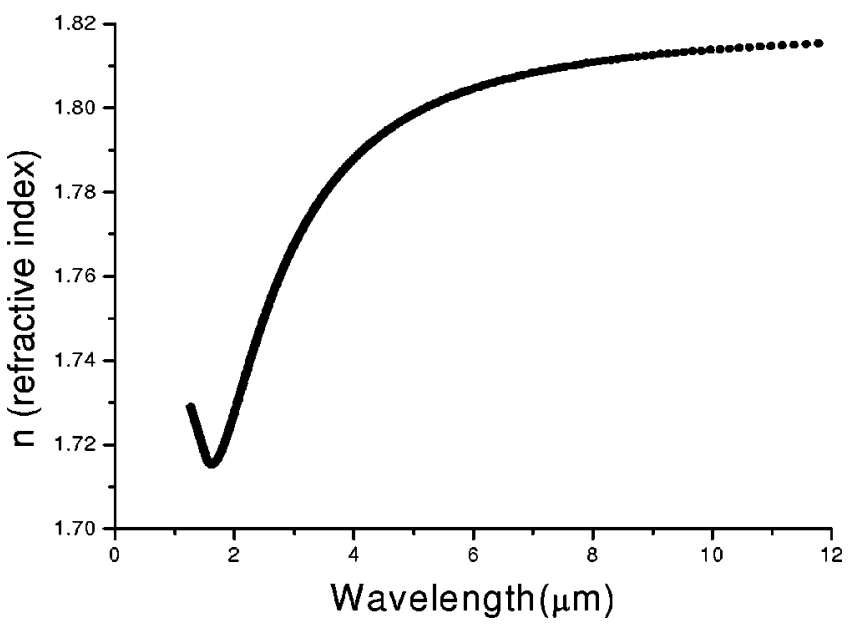

Fig. 6 Refractive index versus wavelength for rf-sputtered $\mathrm{NiO}$ thin film.

Ultrasonic agitation is used to remove the remaining resist. The MOM diode is then fabricated on the patterned slot using the procedure outlined in design 1 .

\subsection{MOM Diode Fabrication Process}

The device in design 1 is fabricated on a Si substrate coated with a layer of $\mathrm{SiO}_{2}$ for electrical isolation. The bottom MOM diode electrode and an IR dipole antenna arm are defined in electron-beam resist PMMA along with one bond pad. A Ni layer of $140 \mathrm{~nm}$ was then deposited using dc magnetron sputtering. The liftoff process is used to remove resist and excess metal. The top MOM diode electrode along with the other IR antenna arm and the second bond pad are patterned, a $\mathrm{NiO}$ layer of $3.5 \mathrm{~nm}$ is then deposited using rf diode sputtering, followed by another 140-nm Ni layer deposited using dc magnetron sputtering, and liftoff is again used to remove resist and excess metal. The refractive index for the NiO layer was determined using an IR ellipsometer, as shown in Fig. 6. The measured refractive index is used to calculate the diode capacitance. Patterning was done by a modified JEOL 5900 scanning electron microscope (SEM) with a $\mathrm{LaB}_{6}$ cathode. The cathode was operated at an accelerating voltage of $30 \mathrm{kV}$. The exposure current was $50 \mathrm{pA}$. Raith Elphy Quantum software was used to manipulate the electron beam. A bilayer resist was used. The bottom layer is a $350 \mathrm{~nm}$ of P(MMA-MAA) 9\% baked for $10 \mathrm{~min}$ on a hotplate at $180^{\circ} \mathrm{C}$. The top layer is $150 \mathrm{~nm}$ of 495-K PMMA baked for $10 \mathrm{~min}$ on a hotplate at $180^{\circ} \mathrm{C}$. The electrodes were exposed as single pixel lines in a $100 \times 100-\mu \mathrm{m}$ writefield at a dose of $15000 \mu \mathrm{C} \mathrm{cm} / \mathrm{s}$ and $61.4 \mu$ s of dwelltime. The resist was postexposure developed for $30 \mathrm{~s}$ in 1:3 mixture of methylisobutylketone:isopropanol (MIBK:IPA). The linewidth of the diode electrodes was controlled by changing electron-beam working distance (WD). In design 1, the exposure was made at a WD of $10 \mathrm{~mm}$, resulting in 250-nm electrodes; and for design 2, 120-nm electrodes were achieved at a WD of 7 $\mathrm{mm}$. 

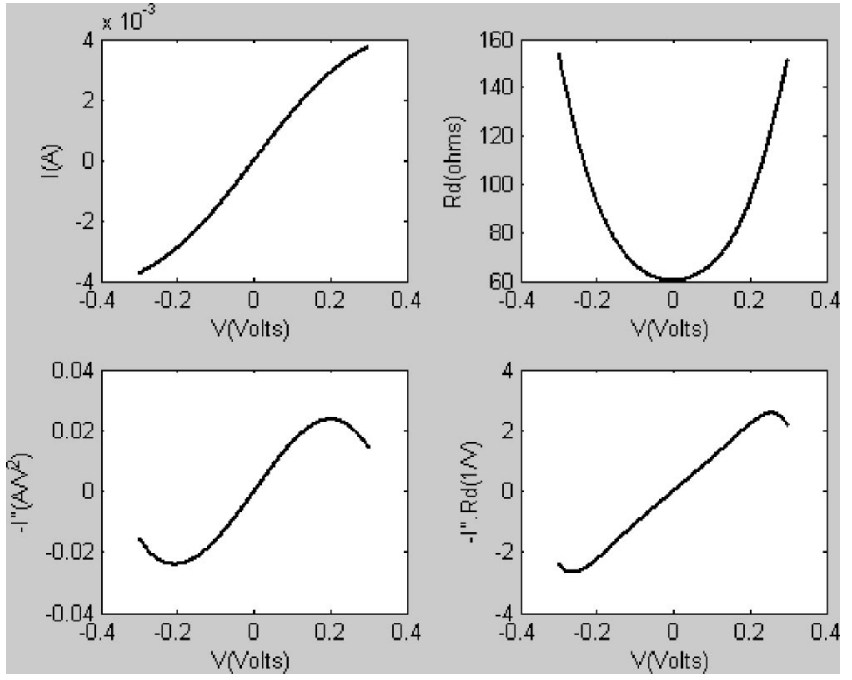

Fig. 7 I-V characteristic curve, its first derivative (Rd), second derivative $\left(-I^{\prime \prime}\right)$ and sensitivity $\left(-l^{\prime \prime} \cdot \mathrm{Rd}\right)$ versus bias voltage for $\mathrm{Ni}$ $\mathrm{NiO}-\mathrm{Ni}$ diode (design 1).

\section{Experimental Results}

\subsection{I-V Characterization for MOM Diodes}

I-V plots for the devices were measured using a parameter analyzer. The captured data for the I-V characteristic curve were further processed to plot the first and second derivatives of the I-V curve and the sensitivity of the device, defined in Eq. (4). Typical plots are displayed in Figs. 7 and 8 for designs 1 and 2, respectively. The diodes in design 1 measured $179 \Omega$ of zero bias resistance; the contact leads connecting the diode to the bond pads are expected to have a contribution to the resistance of the diode. This problem is eliminated in design 2, where the diode is directly contacted to the bond pads and it measured $61 \Omega$ of zero bias

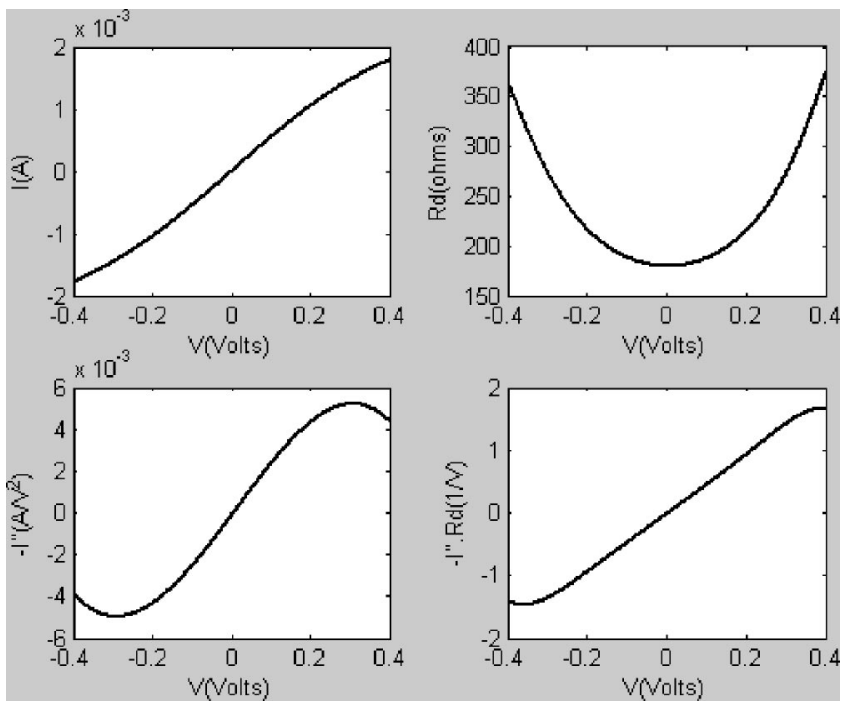

Fig. 8 I-V characteristic curve, its first derivative (Rd), second derivative $\left(-l^{\prime \prime}\right)$ and sensitivity $\left(-l^{\prime \prime} \cdot \mathrm{Rd}\right)$ versus bias voltage for $\mathrm{Ni}$ $\mathrm{NiO}-\mathrm{Ni}$ diode (design 2). resistance. Maximum sensitivity measured was $1.65 \mathrm{~V}^{-1}$ for devices in design 1 and $2.75 \mathrm{~V}^{-1}$ for devices in design 2.

\subsection{Noise in MOM Diodes}

Noise measurements are made to examine the sources of noise in MOM diodes. To determine whether the devices are operating within the Johnson noise limit, noise spectra were captured for a MOM diode and for a resistor having similar dc resistance. The results shown in Fig. 9 indicate the presence of a $1 / f$ noise component for the device. This component could be due to material structures and deposition techniques; in addition, it could be caused by hot electron traps and pinholes in small area devices.

An area study was performed where MOM diodes with 1 - and $0.0625-\mu \mathrm{m}^{2}$ junction areas were fabricated. The captured noise spectra shown in Fig. 10 illustrate higher noise for the diode with smaller junction area. The noise increases at low frequencies and so confirms the presence of higher $1 / f$ noise with smaller junction areas. MOM diode resistance increases as the junction area decreases, matching of the diode to the antenna is therefore affected by changing the junction area. A compromise between matching to the antenna and having lower $1 / f$ noise is required to optimize device performance.

\subsection{Antenna Receiving Properties}

MMW and IR slot antennas are characterized for polarization sensitivity and reception patterns. For testing in the gigahertz region, radiation from a 90 to $94-\mathrm{GHz}$ Gunn oscillator output power was input into a $p-i-n$ diode switch that is used to modulate the MMW power output, and finally a horn antenna was used to irradiate the device. For testing terahertz response, the detector was irradiated by a $\mathrm{CO}_{2}$ laser at $10.6 \mu \mathrm{m}$. The laser radiation is attenuated by a linear polarizer. The polarization incident on the detector is controlled by a half-wave plate. An afocal system is used to expand and collimate the beam; and a final $F / 1$ lens is used to focus the beam into a diffraction-limited spot onto the device. The device response was optimized by accurately positioning it in the focus of the laser beam by mounting it on a three-axis micropositioning stage. A mechanical chopper was used to modulate the laser beam. A lock-in amplifier is used to capture the modulated detector output signal.

For MMW polarization sensitivity measurements, a flexible twistable waveguide was used. The waveguide was connected between the MMW Gunn oscillator and the horn antenna. The waveguide was then twisted at 45-deg increments to modify the radiation incident on the detector. For IR polarization sensitivity measurements, a linear polarizer was fixed and a half-wave plate was rotated to modify the radiation incident on the detector. The polarization ratio was calculated by dividing the detector maximum response by minimum response. The polarization ratios were found to be 13:1 and 4:1 for MMW twin-slot and IR slot antennas, respectively. The dc bias cuts that exist in all the antenna designs in this paper can lower the antenna polarization ratios. The dc bias cuts are $\sim 1.5 \mathrm{~mm}$ each for the MMW slot antenna, $\sim 1.1 \mathrm{~mm}$ each for the MMW twin-slot antenna, and $\sim 12.5 \mu \mathrm{m}$ each for the IR antenna. Being of comparable dimensions to the antennas and are oriented 


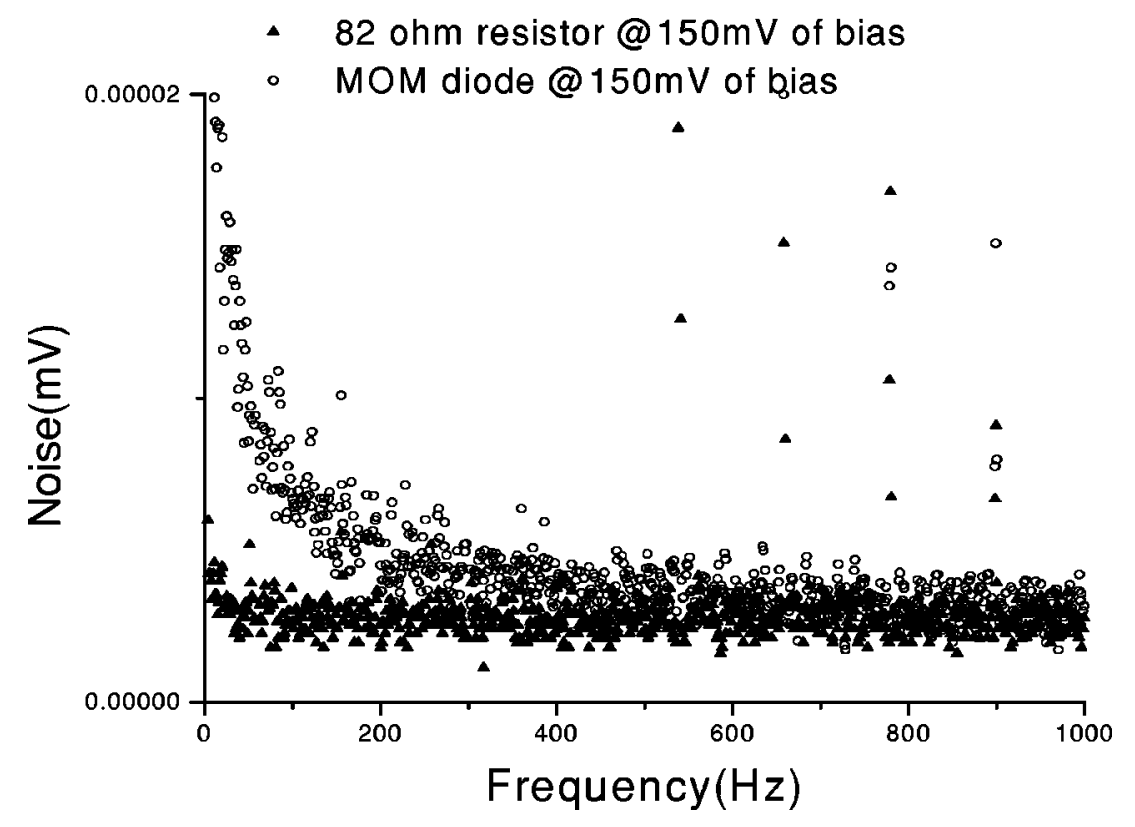

Fig. 9 Noise spectra comparing a MOM diode and an $82-\Omega$ resistor, both at $150 \mathrm{mV}$ of bias.

orthogonally, the dc bias cuts will act as slot antennas and pickup some of the incident radiation that is polarized parallel to the detector, thus reducing the polarization sensitivity of the antennas.

The reception patterns are measured by mounting the detector on a rotational stage. The detector response is measured at 2-deg angular increments of the rotational stage. The $F / 8$ optics with a $200-\mu$ m-diam laser beam spot was used to measure IR patterns. A horn antenna was used to irradiate the detector for MMW antenna reception patterns. The normalized detected voltage in decibels was then plotted versus the angle of incidence. Figure 11 shows the Eand H-plane patterns for a MMW twin slot antenna. The E-plane pattern is broader than the $\mathrm{H}$-plane pattern and both patterns exhibit a single major lobe behavior. The pat-

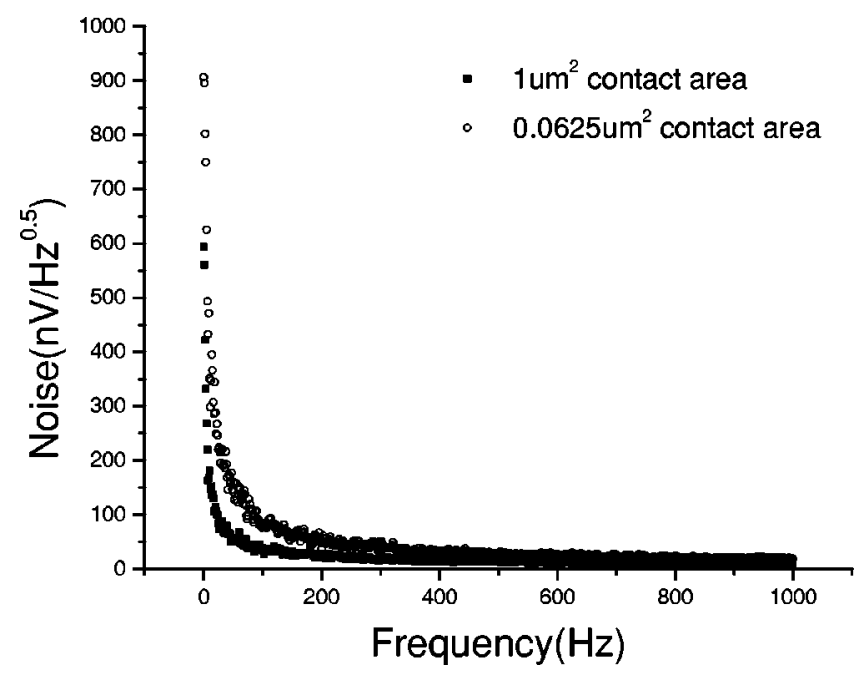

Fig. 10 Noise spectra comparing diodes with 1 - and $0.062-\mu \mathrm{m}^{2}$ junction areas. terns for the IR slot antenna are displayed in Fig. 12. The E-plane pattern is broader than the H-plane pattern.

\subsection{Detector Performance}

Figures 13 and 14 show plots for the normalized MMW and IR responses and the second derivative of the I-V characteristic curve versus bias voltage of the diode. It is observed that both MMW and IR voltage responses in design 2 follow the second derivative of the I-V curve more closely than the voltage responses in design 1. Equation (3) states that the tunneling effect is responsible for the fact that the voltage response of an antenna-coupled MOM diode is proportional to the second derivative of the I-V curve. Therefore, it could be said that the response of devices in design 2 shows a more dominant tunneling mechanism when compared to devices in design 1 , a behavior that could be explained by the fact that the measured I-V characteristics for the diode in design 1 describe the combined behavior of the diode and the contact lead resistances.

Noise equivalent power (NEP) is the amount of radiant power collected on a detector that will produce an SNR of 1 (Ref. 10). NEP is given by

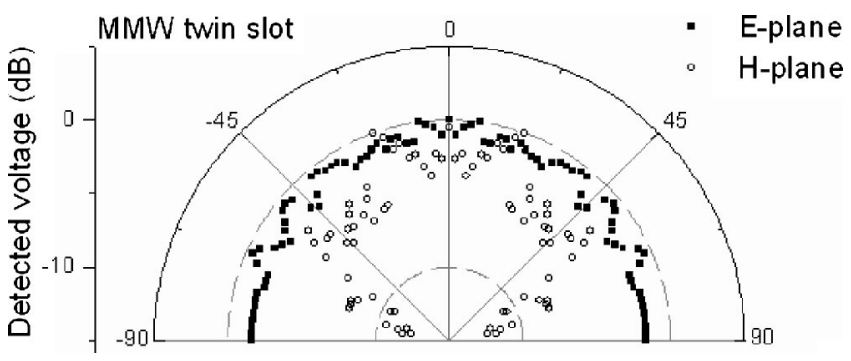

Fig. 11 E- and H-plane patterns for MMW twin-slot antenna. 


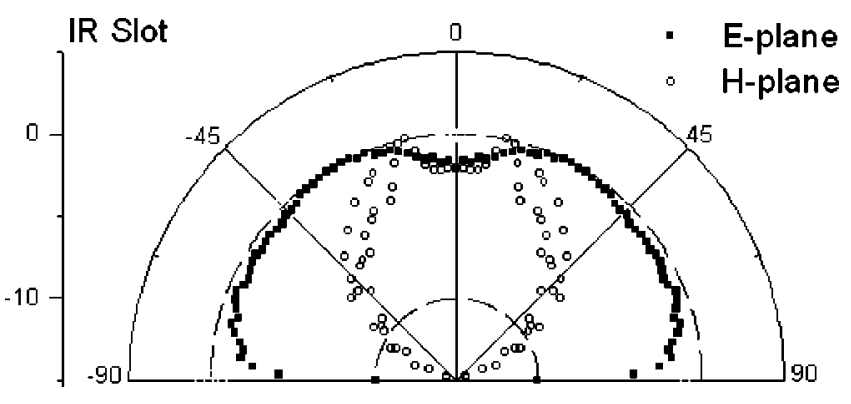

Fig. 12 E- and H-plane patterns for IR twin-slot antenna.

$\mathrm{NEP}=\frac{\phi_{i n}}{\mathrm{SNR}}$

where $\varphi_{\text {in }}$ is the flux incident on the detector and is given by

$\phi_{\mathrm{in}}=E A_{\mathrm{det}}$,

where $E$ is the irradiance, $A_{\text {det }}$ is the optically active detector area, and SNR is the signal-to-noise ratio. The MMW irradiance is the power contained in the MMW beam radiated from the horn antenna. The beam is diverging at an angle of $25 \mathrm{deg}$. The IR irradiance $E$ is the irradiance of a diffraction-limited spot of produced by an $F / 1$ optical train; the spot radius is approximately $12 \mu \mathrm{m}$. The optically active detector area $A_{\text {det }}$ is approximated to be the actual physical area of the antennas. The best achieved NEPs are 50 and $72 \mathrm{pW} / \mathrm{Hz}^{0.5}$ in the MMW and IR, respectively. The measured and calculated results are displayed in Table 1. For simultaneous response verification, MMW and IR radiation were modulated at 3 and $2.6 \mathrm{kHz}$, respectively, and made simultaneously incident on the device. Simultaneous response was demonstrated..$^{5}$

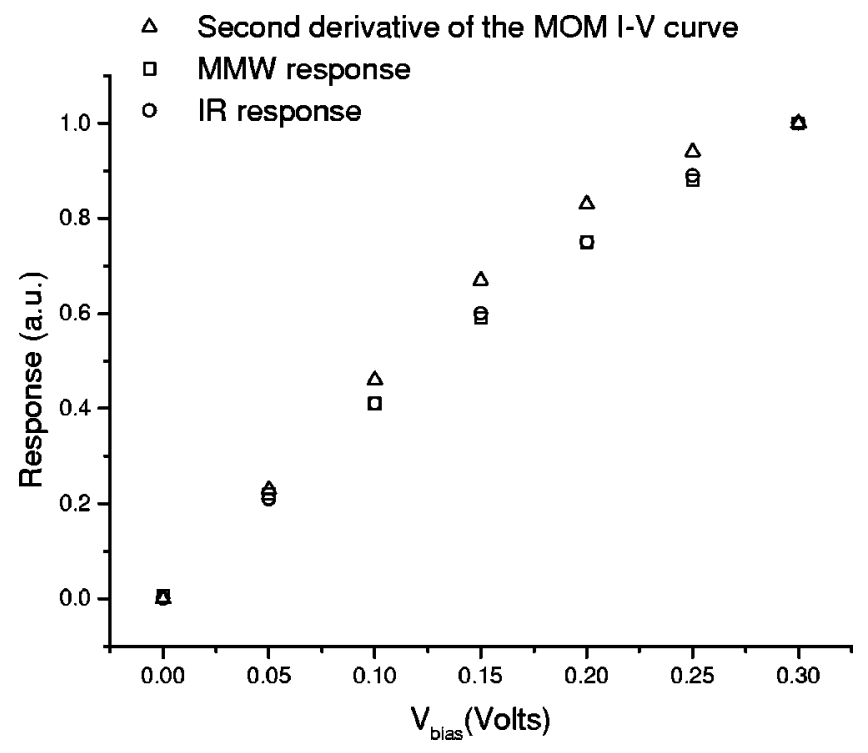

Fig. $13 \mathrm{MMW}$ and IR responses and the second derivative of the $\mathrm{I}-\mathrm{V}$ characteristic curve (arbitrary units) versus diode bias (design 1).

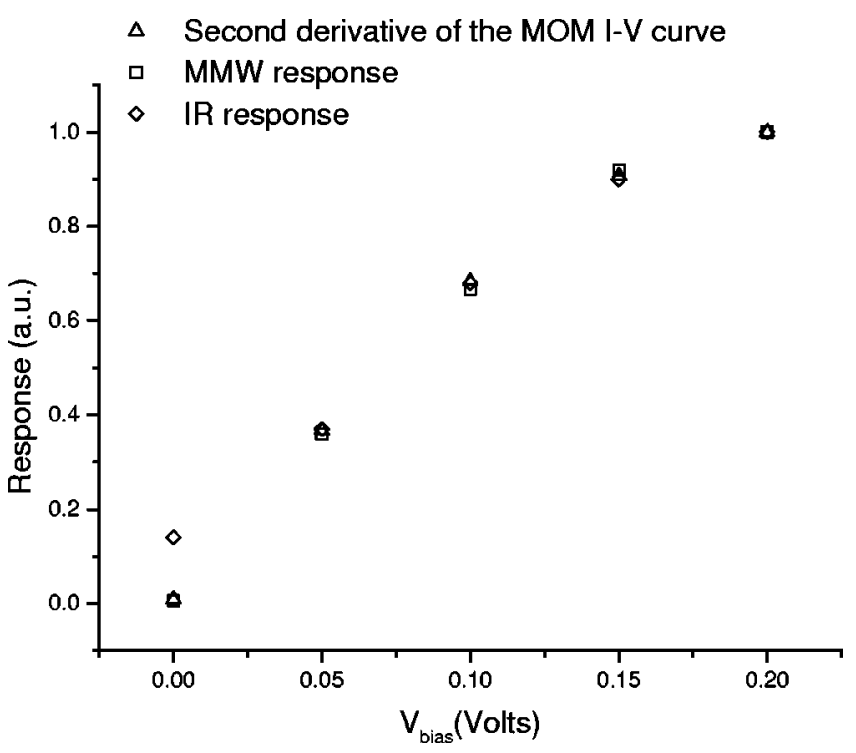

Fig. $14 \mathrm{MMW}$ and IR responses and the second derivative of the $\mathrm{I}-\mathrm{V}$ characteristic curve (arbitrary units) versus diode bias (design 2).

\section{Conclusions and Future Work}

The MMW response was improved by implementing the CPW-fed twin slot configuration as opposed to the singleslot configuration. The improvement was mainly caused by reducing power coupled to surface waves, and also due to proper matching between the diode and the antenna.

The IR response showed better performance when the dipole was used as opposed to the slot antenna configuration. Both configurations are predicted to have good matching between the diode and the antenna. The better response in the dipole configuration could be caused by lower noise due to larger diode contact area. The IR response can be improved by using a CPW-fed twin-slot antenna, which has a better feed configuration; in addition, a superstrate could also be used for better absorption of IR radiation.

More noise measurements must be made for better understanding for the origin of the noise in the devices. The fabrication process also must be improved to achieve better film quality. Currently, all our films are deposited at chamber base pressures of $5 \times 10^{-6}$ torr; high-quality films require lower chamber pressures.

Table 1 Irradiance, optically active detector areas, SNR, and NEP for the detectors.

\begin{tabular}{lcll}
\hline \hline & & Design 1 & Design 2 \\
\hline \multirow{2}{*}{$E\left(\mathrm{~W} / \mathrm{m}^{2}\right)$} & MMW & $9.8 \times 10^{0}$ & $4.5 \times 10^{0}$ \\
& $\mathrm{IR}$ & $1.4 \times 10^{6}$ & $3.6 \times 10^{6}$ \\
$A_{\text {det }}\left(\mathrm{m}^{2}\right)$ & $\mathrm{MMW}$ & $9.7 \times 10^{-9}$ & $1.1 \times 10^{-7}$ \\
& $\mathrm{IR}$ & $7.5 \times 10^{-13}$ & $7.2 \times 10^{-12}$ \\
SNR & $\mathrm{MMW}$ & $1.1 \times 10^{3}$ & $1.0 \times 10^{4}$ \\
& $\mathrm{IR}$ & $1.5 \times 10^{4}$ & $4.2 \times 10^{4}$ \\
\multirow{2}{*}{$\mathrm{NEP}\left(\mathrm{W} / \mathrm{Hz}^{0.5}\right)$} & $\mathrm{MMW}$ & $86 \times 10^{-12}$ & $50 \times 10^{-12}$ \\
& $\mathrm{IR}$ & $72 \times 10^{-12}$ & $617 \times 10^{-12}$ \\
\hline \hline
\end{tabular}




\section{Acknowledgment}

This work was supported by Defense Advanced Research Projects Agency/Army Research Office (DARPA/ARO) under Contract No. DAAD19-02-1-0232.

\section{References}

1. C. Fumeaux, W. Herrmann, F. K. Kneubühl, and H. Rothuizen, "Nanometer thin-film Ni-NiO-Ni diodes for detection and mixing of 30 THz radiation," Infrared Phys. Technol. 39, 123-183 (1998).

2. J. W. Dees, "Detection and harmonic generation in the sub-millimeter wavelength region," Microwave J. 9, 48-55 (1966)

3. A. Sanchez, C. F. Davis, Jr., K. C. Lui, and A. Javan, "The MOM tunneling diode: theoretical estimate of its performance at microwave and infrared frequencies," J. Appl. Phys. 49, 5270-5277 (1978).

4. B. M. Kale, "Electron tunneling devices in optics," Opt. Eng. 24, 267-274 (1985).

5. M. R. Abdel-Rahman, F. J. González, and G. D. Boreman, “Antennacoupled metal-oxide-metal for dual-band detection at $92.5 \mathrm{GHz}$ and 28 THz," IEE Electron. Lett. 40, 116-118 (2004)

6. G. Eleftheriades and G. Rebeiz, "Self and mutual admittance of slo antennas on a dielectric half-space," Int. J. Infrared Millim. Waves 14 1925-1946 (1993)

7. Zeland Software Inc., IE3D simulator (2004).

8. D. B. Rutledge, D. P. Neikirk, and D. Kasilingam, "Integrated-circui antennas," in Infrared and Millimeter Waves, Vol. 11, Millimeter Components and Techniques, Part II, K. J. Button, Ed., pp. 1-90, Academic Press, New York (1984).

9. Y. Abe and Y. Yasuoka, "Operating properties of thin-film slot antennas at 2.5-THz submillimeter wave band," Electron. Commun. Jpn. 85, 8-15 (2002).

10. E. L. Dereniak and G. D. Boreman, "Figures of merit for optical detectors," in Infrared Detectors and Systems, J. W. Goodman, Ed. pp. 200-229, Wiley, New York (1996).

Mohamed R. Abdel-Rahman received the BSc in physics from the American University in Cairo, Egypt, in 1999 and the MSc and PhD in electrical engineering from the University of Central Florida in 2002 and 2004, respectively. He worked as a graduate research assistant in the Infrared Systems Laboratory at the College of Optics and Photonics (CREOL) from 2000 until 2004. His research interest is in the area of antenna-coupled detectors for millimeter-wave and infrared focal plane arrays. He is a member of SPIE and IEEE.

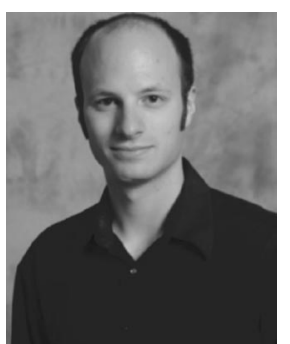

Brian Monacelli received the BS degree in applied optics at Rose-Hulman Institute of Technology, Terre Haute, Indiana, in 2000 and the MS degree in optics from the Institute of Optics, University of Rochester, in 2001. He is currently a graduate research and teaching assistant while working toward the PhD in optics at the University of Central Florida. His research interests include infrared frequency selective surfaces, uncooled antenna-coupled infrared detectors, and infrared system design. He has worked as a research scientist with Rocketdyne Boeing Lasers (Electro-Optics Systems Division), Silicon Valley Group (Lithography Division), and NSWCCrane. Mr. Monacelli is a student member of the Optical Society of America, the International Society for Optical Engineering, and the IEEE. He currently serves as president of the UCF Student Chapter of the Optical Society of America.

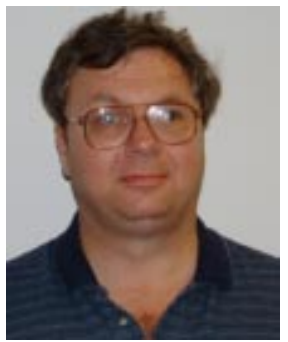

Arthur R. Weeks received his $\mathrm{PhD}$ degree in electrical engineering from the University of Central Florida in Orlando in 1987. After completion of his PhD, he spent one year at the Royal Signals and Radar Establishment in Malvern, England, studying laser beam propagation. Dr. Weeks just recently left his vice president of corporate technology position at Invivo Research Inc. to join the Electrical Engineering Department at the University of Central as a visiting associate professor. Dr. Weeks' interest include color image processing techniques, the reduction of noise within images using adaptive nonlinear filters, and the use of artificial neural networks in pattern recognition. Dr. Weeks is a member of the IEEE, SPIE, and Tau Beta Pi.

Guy Zommo: Biography and photograph not available.

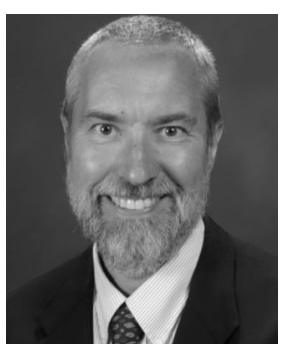

Glenn D. Boreman is the Trustee Chair Professor of Optics, Electrical Engineering, and Physics at the College of Optics and Photonics (CREOL) at the University of Central Florida. He received his BS degree from the Institute of Optics, University of Rochester, and his PhD from the Optical Sciences Center, University of Arizona. He has been a visiting scholar at Imperial College in London, the Swiss Federal Institute of Technology (ETH) in Zürich, and the Defense Research Agency (FOI) in Linköping, Sweden. Dr. Boreman is currently the editor-in-chief of OSA's Applied Optics, and is a past member of the SPIE Board of Directors. He is coauthor of the graduate textbook Infrared Detectors and Systems, author of Modulation Transfer Function in Optical \& Electro-Optical Systems, and Basic Electro-Optics for Electrical Engineers. He has published more than 100 papers in the areas of IR detector and focal-plane analysis, optics of random media, IR scene projection, and transfer-function techniques. Dr. Boreman is a fellow of SPIE and OSA and he and two of his students received the 1995 Kingslake Medal from SPIE. 